\title{
Huge Shape Recovery of the Knitting actuators made by TiNi Shape Memory Alloy Wires
}

\author{
Miu Hatamura ${ }^{1 *}$ and Taishi Wada ${ }^{2}$ \\ 1 Yokohama National University, Yokohama Japan \\ 2 Kyoto University, Kyoto, Japan \\ * Corresponding author: Fax: +81-045-339-4099, and/or e-mail:hatamura-miu-hz@ynu.jp
}

\begin{abstract}
Shape recovery effect of the specimens made by knitting of a TiNi shape memory alloy wire has been investigated. Since there are hundreds of knitting method in the knitting world, we selected the main two methods of knitting for fabrication of the specimen, i.e., Stockinet knitting and Garter knitting, and made tensile tests to get the mechanical properties of these specimens experimentally. These specimens are made of SMA wire of $0.3 \mathrm{~mm}$ diameter. The experimental results show that the maximum of the output strain is more than $100 \%$ and the maximum of the output stress is $1.8 \mathrm{MPa}$.

Key words: Knitting, TiNi, Shape Memory Alloy
\end{abstract}

\section{INTRODUCTION}

In recent years, as advance of the technology, the research of the materials which properties autonomously change depending on the surrounding environment is carried out flourishingly. These are called intelligent materials or smart materials. Development of the machine system using such materials attracts much attention. One of the materials which has large advances most in the intelligent material is shape memory alloys (SMAs). Since shape memory effect was discovered in TiNi-SMA in 1959, various studies on SMAs have been performed [1]. In 1969 , the first practical application was the pipe coupler for a U.S. Navy F-14 fighter aircraft [2]. At the present passing more than half a century after it was discovered, the development of the actuator using the SMAs is carried out flourishingly and the practical use advances as intelligent metal materials in various fields, such as automotive, aerospace, robotics and biomedical, using the high mechanical characteristics, i.e., the stability of the shape memory effect or ductility, the strength, corrosionresistant $[3,4,5]$. We can say almost only one demerits of SMAs is the maximum output strain is limited to approximately $5 \%[6,7]$. To obtain large output strain, the technique of working into a coil is adopted usually. By this technique, recent research have shown that SMA actuators can provide an opportunity to replace electric motors and pneumatic actuators. However, the coil made by SMAs reduces the output stress remarkably. If we increase the wire diameter, we can suppress the decrease in this output stress, however, it leads to a decrease in operation speed [7].

In recent years some papers reported that large strain can be provided by the knitting into stitch

structure. The technique of knitting into stitch structure brings the large strain caused not only by bending of SMA wire but also by slipping peculiar to stitch structure. That technique is expected to increase its output strain largely $[7,8,9]$. In this study, we shows the preliminary experiment using the specimen made by hand-knitted with $0.15 \mathrm{~mm}$ diameter TiNi wire. As a result of that, remarkably huge reversible strain (over $500 \%$ ) are observed. By the way, the difference in output strain and stress by the stitch structure is little known. Therefore the main purpose of this study is to investigate the difference in output strain and stress experimentally of the different kitting specimens. Since there are hundreds of knitting method in the knitting world, we selected the main two methods of knitting for fabrication of the actuator, such as, Stockinet knitting and Garter knitting, and compared the mechanical properties of these actuators experimentally.

\section{EXPERIMENTAL}

\subsection{Basic structure of kitting specimens}

Figure 1 (a) and (b) show the schematic drawings of a stitch structure of the stockinet stitch and the garter stitch, respectively. Wale-direction and course-direction are also shown in Figure 1. General knitting is constructed by a wire without interruption consecutively and comprised of series of unit cell shown in Figure 2. One unit cell has one loop of the arc type and two legs. A loop interlock with two legs of the previous row and the legs of the unit cell interlock with the loop of the following row. The stitch structure is distinguished by the position of the loops and the legs of the unit cells with the previous row. The stitch that a loop of the unit cell is placed in front of legs of the unit cell of the previous row is called knit loop. On the other hand, the stitch that a loop of the unit cell is placed in the rear of legs of the unit cell of the previous row is called purl loop. In the stockinet stitch that is the most common stitch structure all loops of the unit cell are placed in front of the unit cell of the previous row, i.e. the stockinet stitch is comprised of the series of knit stitches. The garter stitch that is generally high in elasticity is comprised of alternating rows of knit and purl loops. In this paper the number of unit cell of the wale direction and the number of unit cell of the course direction are defined as $m$ and $n$, respectively. 


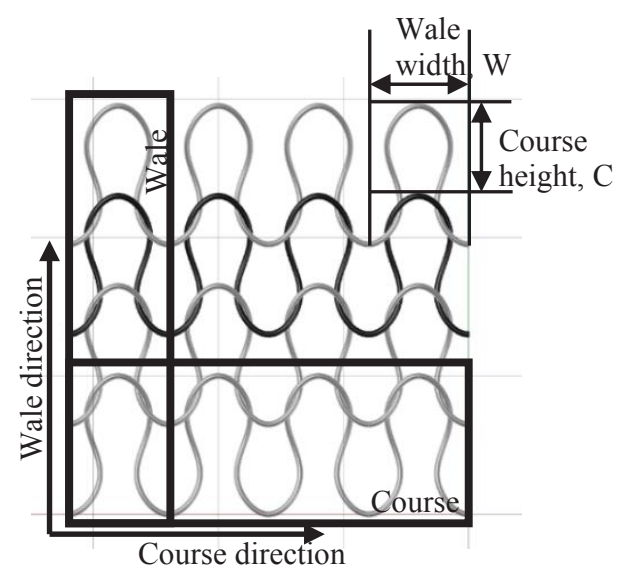

Figure 2(a). The stockinet stitch.

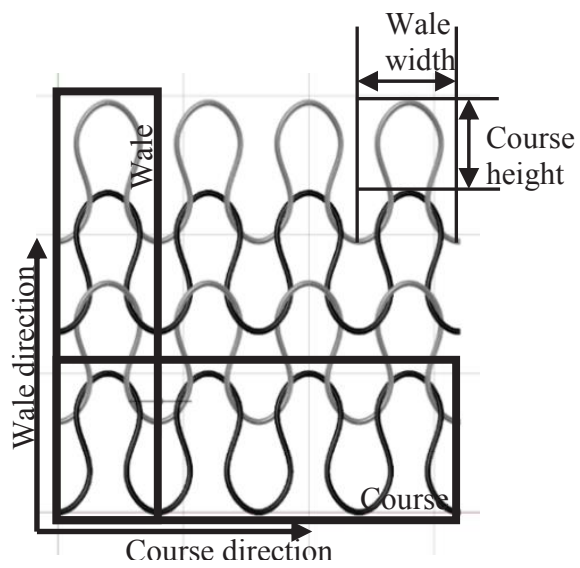

Figure 1(b). The garter stitch.

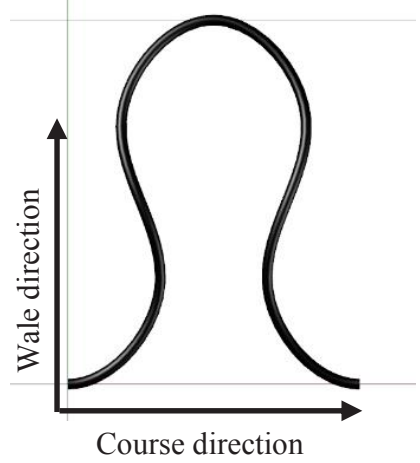

Figure 2. The unit cell in the stitches.

2.2 Preliminary experimental

Preliminary experimental was performed using the specimens made by knitting $0.15 \mathrm{~mm}$ diameter wire (Af : $363 \mathrm{~K})$. The four kind of knitting way, i.e., Stockinet, Reverse stockinet, Garter stitch and Rib stitch, are used to manufacture the specimens. The microscopical view of the specimen is shown in Figure 3. The TiNi wire was shapememorized in a shape of line and knitted as shown in Figure 3. Figure 4 shows the Garter stitch specimen, which was pulled huge strain, such as $630 \%$, and showed perfect recovery to the original shape due to heating up to the transformation temperature. This should be champion data
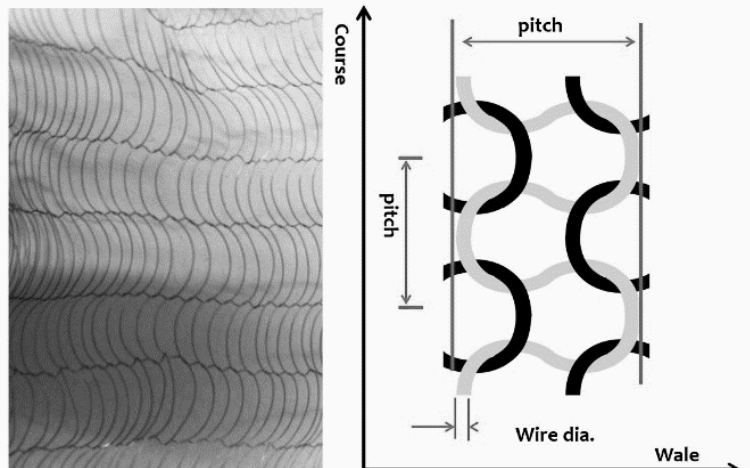

Figure 3. The kitting specimen with $0.15 \mathrm{~mm}$ wire.

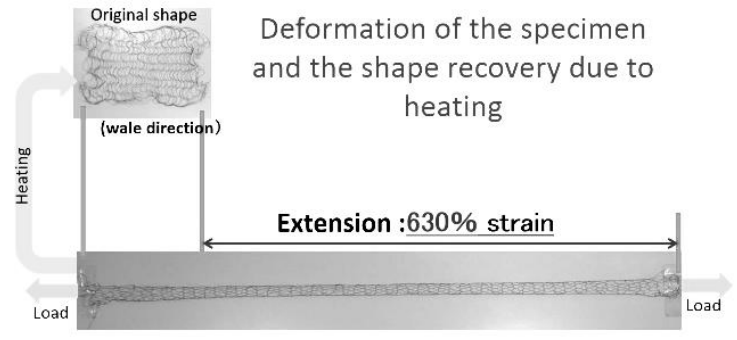

Figure 4. Deformation of the Garter stitch specimen and the shape recovery due to heating. The TiNi wire with diameter of $0.15 \mathrm{~mm}$ are shape memorized in a shape of line and knitted as shown in Figure 3.

of the TiNi structural specimens. Mechanism of the Shape recovery of the Knitting specimen made by a TiNi wire with diameter of $0.15 \mathrm{~mm}$ is shown in Figure 5. When the specimen are loaded, it elongated over $500 \%$ due to the shape change of the loops, i.e., unit cell in the stitches. Due to further loading, the elongation of specimen occurs due to the phase change of the TiNi wire. By heating, the phase of TiNi wire return to the parent phase and the shape change of the loops occurs. As a result, the shape of specimen return to the original

Experimental results of the performance curves of the knitting specimen made by a TiNi wire with diameter of $0.15 \mathrm{~mm}$, which was shape memorized in a shape of line, are shown in Figure 6. It is noted that the huge output strain and recovery strain are obtained in these knitting specimens. Therefore, it is expected that we can obtain huge strain by knitting and also can use these knitting specimen as actuators with large output stroke and power.

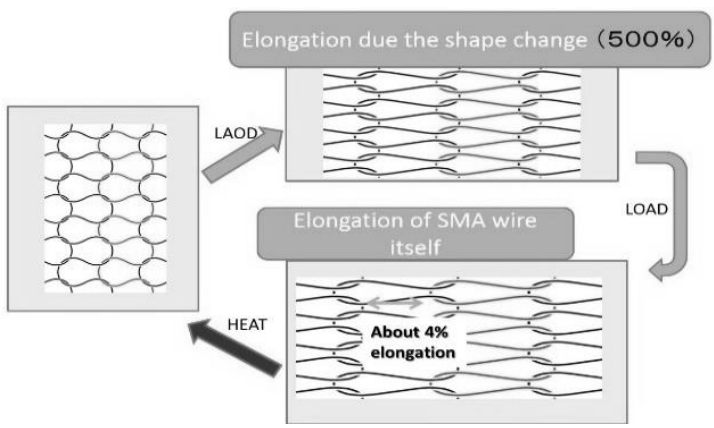

Figure 5. Mechanism of the Shape recovery of the knitting specimen made by a TiNi wire with diameter of $0.15 \mathrm{~mm}$. The wire was shape memorized in a shape of line. 


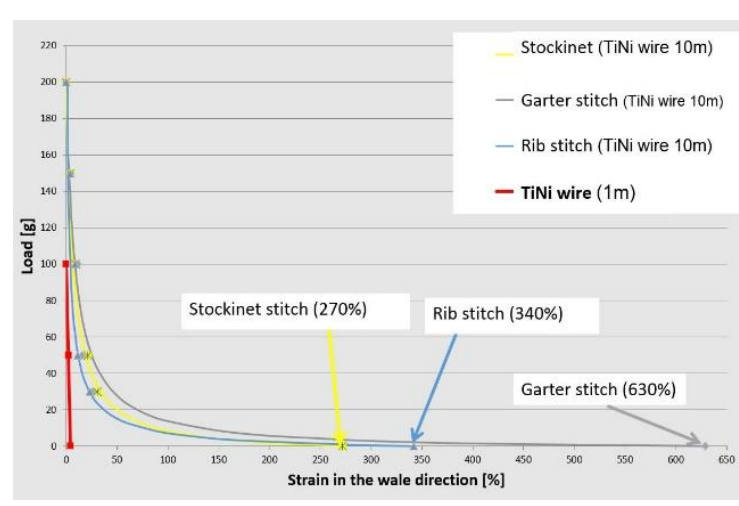

Figure 6. Experimental results of the performance curves of the knitting specimen made by a TiNi wire with diameter of $0.15 \mathrm{~mm}$, which was shape memorized in a shape of line.

\subsection{Experimental setups}

The material used for actuators was a commercial TiNi SMA wire with $0.3 \mathrm{~mm}$ diameter. The composition of the alloy was Ti-49.6 at $\% \mathrm{Ni}$. The temperature of shape memorization heat treatment was $673 \mathrm{~K} 40 \mathrm{~min}$. The transformation temperature (Af) was approximately $330 \mathrm{~K}$. Figure 7 shows the stress-strain curve of the shape memory TiNi alloy wire, which was obtained by the tension-unloading test at room temperature $(288 \mathrm{~K})$. The two types of the knitted specimens, plain loop knit and garter loop knit, were made of the $5 \mathrm{~m}$ wire, using a lilyyarn knitting machine. Figure 8(a) and 8(b) show pictures of the actuator specimens and the schematic drawings of their detailed structures. After the wires were knitted into the loop knit actuator specimens, which were bound in state widened in the cylindrical diameter direction and are dealt with shape memorization heat treatment. Table I shows these geometric parameters of the knitted actuators. The gauge length and cylindrical diameter in the austenite free state are expressed as Lwo and $\mathrm{D}_{0}$, respectively The average course height, $\mathrm{C}_{0}$, was determined by dividing the gauge length by the number of courses, $\mathrm{N}_{\mathrm{C}}$. Similarly, the wale width, $\mathrm{W}_{0}$, was determined by dividing the circumferential length of the cylinder, $\mathrm{L}_{\mathrm{C}}$, by the number

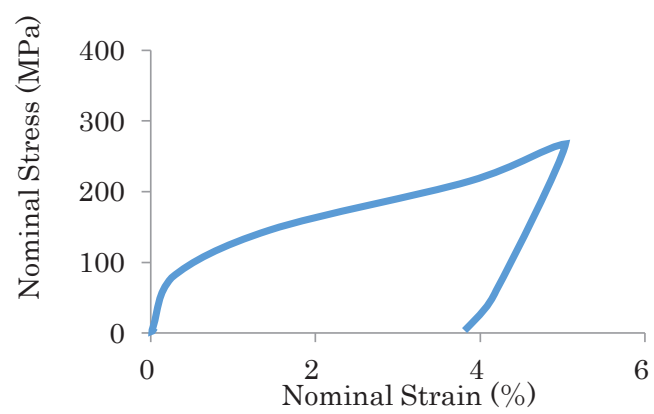

Figure 7. Stress-strain curve of the TiNi wire used for specimens. The wire returns to original shape by heating (a) Plain loop

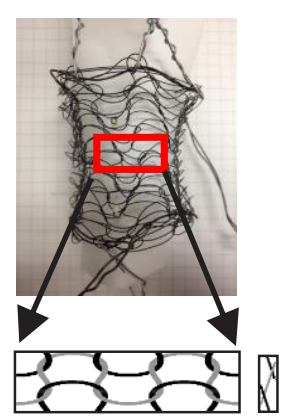

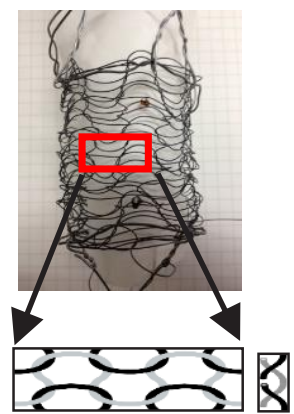

(c) Garter loop
Figure 8 Pictures and schematic drawings of the actuators

of courses, $\mathrm{N}_{\mathrm{W}}$.

The tensile tests were carried out on a uniaxial tensile test machine IS-10T. The load was measured by a load cell LC1205-K050 with a rated capacity of $50 \mathrm{~N}$. The displacement was measured

by a camera for gauge length observation CV-200M and CV-3500. The outputs are monitored and recorded by PCsystem KV5000 and KV COM.

Experimental procedure is as follows: At first, the specimen is tensile until designated load occur. Then, the load is released. Afterward, the specimen is heated to 373 $\mathrm{K}$ with the industrial dryer. The measured load is caused by shape memory effect. The load is released again due to air cooling.

\section{RESULT AND CONSIDERATION}

Figure 10 shows the true stress-strain curves of the Stockinet and Garter actuator specimens. Since the curves were obtained at room temperature under a loading rate of $3.0 \mathrm{~mm} / \mathrm{min}$, the phase of $\mathrm{TiNi}$ is martensite before loading. During loading the shape change by slipping (See

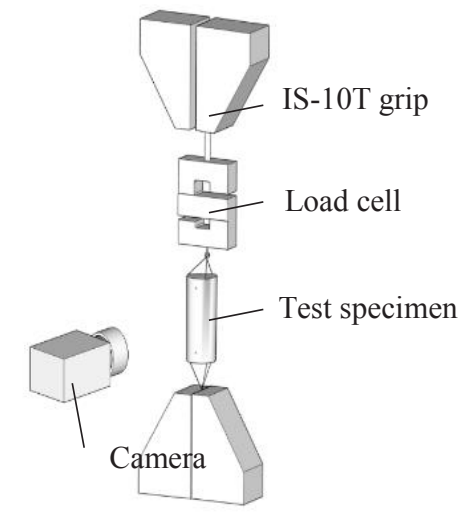

Figure 9. Schematic drawing showing the experimental setup.

Table I . Dimension of the actuator specimens.

\begin{tabular}{|c|c|c|c|c|c|c|c|c|}
\hline & $\mathrm{d}(\mathrm{mm})$ & $\mathrm{N}_{\mathrm{C}}(\mathrm{mm})$ & $\mathrm{N}_{\mathrm{W}}$ & $\mathrm{D}_{0}$ & $\mathrm{~L}_{\mathrm{W} 0}(\mathrm{~mm})$ & $\mathrm{L}_{\mathrm{C} 0}(\mathrm{~mm})$ & $\mathrm{C}_{0}(\mathrm{~mm})$ & $\mathrm{W}_{0}(\mathrm{~mm})$ \\
\hline Stockinet knitting & 0.3 & 6 & 15 & 30 & 30 & 94 & 2.0 & 15.7 \\
\hline Garter knitting & 0.3 & 6 & 15 & 31 & 27 & 97 & 1.8 & 16.2 \\
\hline
\end{tabular}



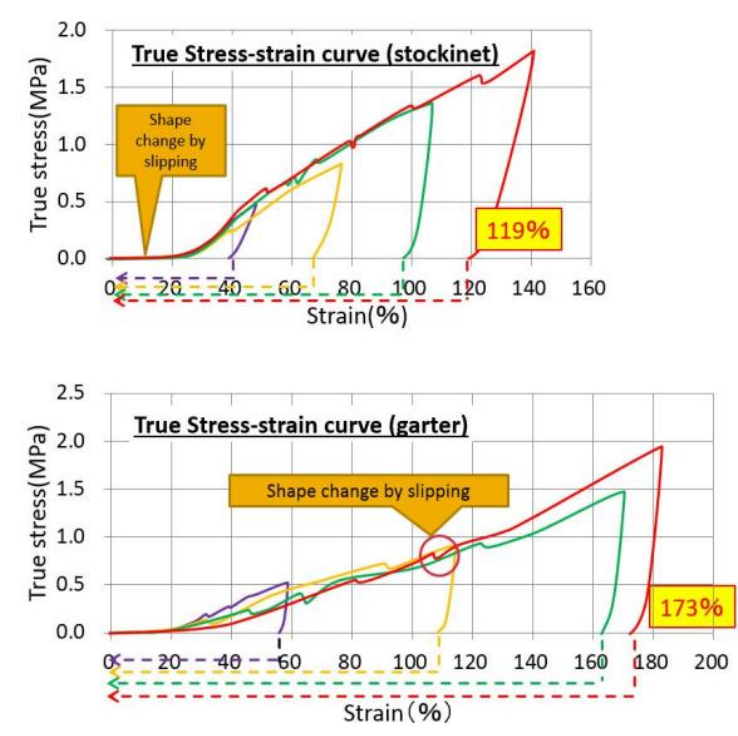

Figure 10. The true stress-strain curves of Stockinet knitting and garter loop knitting actuators.

Figure 11.). For Stockinet actuator specimen, the strain was applied to about $48 \%$ (True stress was about $0.5 \mathrm{MPa}$ ) and recovered to zero due to heating, in the next cycle the strain was applied to about $76 \%$ (True stress was $0.8 \mathrm{MPa}$ ) and recovered to zero due to heating, in the third cycle the strain was applied to about $107 \%$ (True stress was about 1.4 $\mathrm{MPa}$ ) and recovered to zero due to heating, and in the fourth cycle the strain was applied to about $141 \%$ (True stress was about 1.8 $\mathrm{MPa}$ ) and recovered to zero due to heating. The obtained strain of $119 \%$ is almost due to the shape change by slipping, which mechanism is schematically drawn in Figure 11. For Garter actuator specimen, the strain was applied to about $58 \%$ (True stress was about $0.5 \mathrm{MPa}$ ) and recovered to zero due to heating, in the next cycle the strain was applied to about $112 \%$ (True stress was $0.8 \mathrm{MPa}$ ) and recovered to zero due to heating, in the third cycle the strain was applied to about $170 \%$ (True stress was about $1.5 \mathrm{MPa}$ ) and recovered to

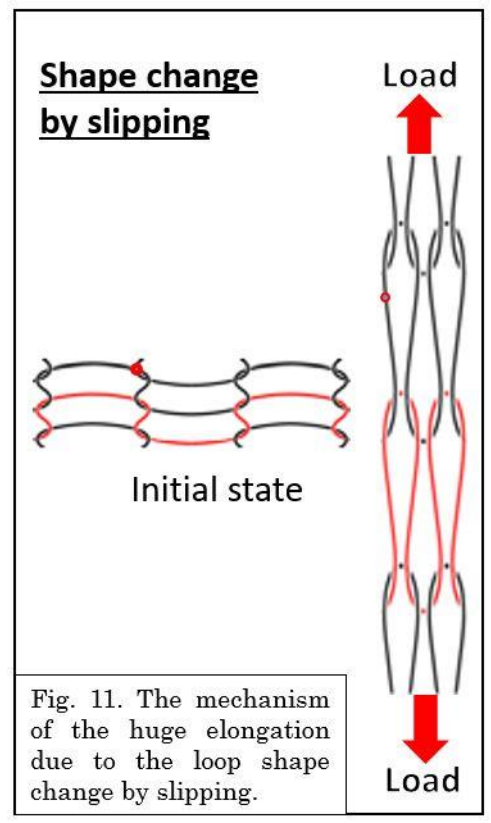

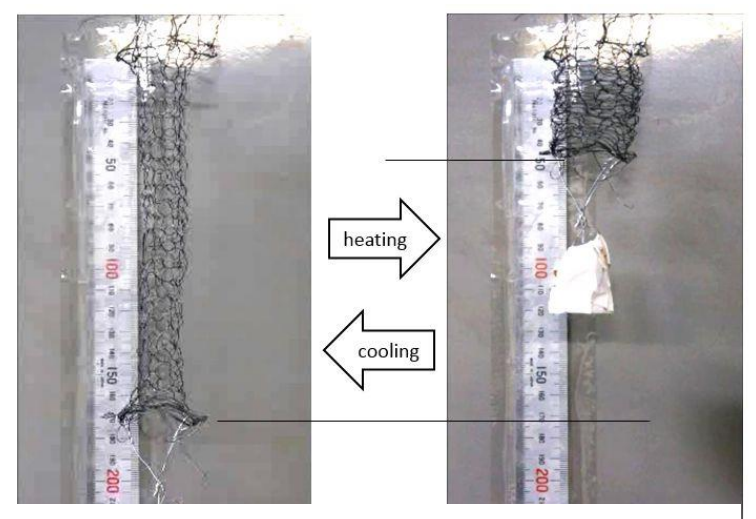

Figure 12. Pulling up a dead load of $5 \mathrm{~N}$ by the garter actuator specimen.

zero due to heating, and in the fourth cycle the strain was applied to about $182 \%$ (True stress was about $1.9 \mathrm{MPa}$ ) and recovered to zero due to heating. The obtained strain of $173 \%$ is almost due to the loop shape change by slipping, which mechanism is schematically drawn in Figure 11.

Important is that the huge strain of the actuator specimens measured is due to the shape change by slipping, as far as our observation. So the key point to get huge strain is which kinds of kitting way are selected and which knitting way can show the large shape change by slipping as shown in Figure 11. Further discussion must be necessary in further studies, which should use a kind of the mathematical science of geometry.

Performance of the specimen as an actuator was investigated. Using the garter cylindrical specimen made of $5 \mathrm{~m}$ TiNi wire of $0.3 \mathrm{~mm}$ wire diameter, the load (5 N) was pulled up by heating with a hair dryer and down by cooling with a hair dryer, which are shown in Figure 12.

Figure 13 shows the output performance of the both actuator specimens, which was obtained by the test shown in Figure 12. It is calculated from this figure that maximum output work is $0.14 \mathrm{Nm}$ and $0.07 \mathrm{Nm}$ for the stockinet and garter, respectively. It is noted that the output performance of these actuator specimens is better than the human muscle, as shown in Figure 13. The maximum work load of stockinet is larger. On the other, Strain of garter knitting is larger than stockinet in the domain of the small stress. The garter stitch has a bigger thickness than

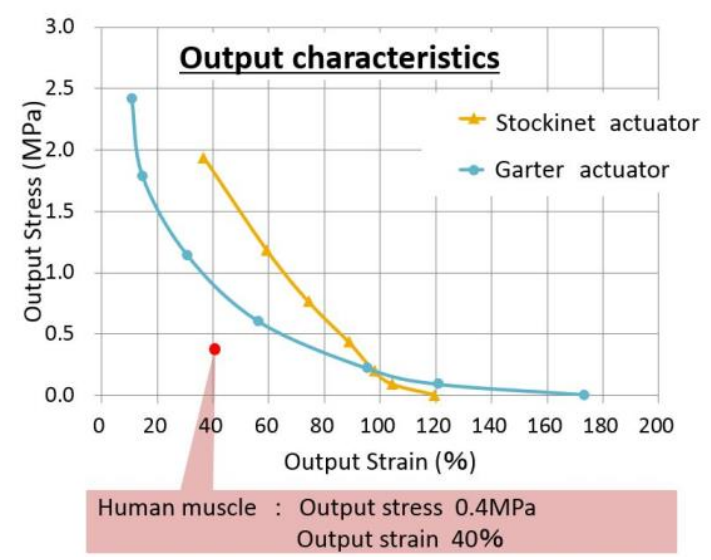

Figure 13. Output performance of the actuator specimens. 
stockinet in the initial state. The thickness difference make the output difference. We can say that it is important to choose how to knit the TiNi wire depending on a purpose of use of this actuator.

\section{CONCLUSION}

(1) New type of TiNi SMA actuators were developed.

(2) The actuator specimen was made from TiNi wires by knitting of them using several kinds of knitting way.

(3) For the stockinet knitting actuator specimens, the maximum output strain is $119 \%$ and the maximum output work is $0.14 \mathrm{Nm}$.

(4) For the garter knitting actuator specimens, the maximum output strain is $173 \%$ and the maximum output work is $0.07 \mathrm{Nm}$.

(5) Huge strain was observed in these specimens.

(6) Output characteristics of the specimens are different from how to knitting.

Acknowledgments

This work was supported in part by Grants-in-Aid for Specially Promoted Research (No. 25000012), Young Scientists Research (A) (No. 24686018), and Challenging Exploratory Research (No. 26630009) of the Japan Society for the Promotion of Science, Japan.

\section{References}

[1] J. M. Jani, M. Leary, A. Subic, and M. A. Gibson, Materials and Design, Vol. 56, 1078-1113 (2014).

[2] G. B. Kauffman, and I. Mayo, The Chemical Educator, Vol. 2, 1-21 (1997)

[3] M. H. Wu, and L. M. Schetky, International conference on shape memory and superelastic technologies, Pacific Grove, California, 171-182 (2000)

[4] D. Hartl, and D. C. Lagpudas, Proceedings of the institution of Mechanical Engineers, Part G: Journal of Aerospace Engineering, Vol.221, 535-552 (2007)

[5] L.G. Machado, and M.A. Savi, Brazilian Journal of Medical and Biological Research, Vol. 36, 683-691 (2003)

[6] K. N. Melton, "Shape Memory Materials", Ed. by K. Otsuka, and C. M. Wayman, Cambridge University press, Cambridge (1999) pp. 220-239.

[7] S. Dono, A. Saito, and T. Kuwata, Knit Structure SMA Actuator for Wearable Artificial Muscle Systems, Matsushita Electric Works Technical Report, (Aug. 2003)

[8] T. Saito, Q. Ji, K, Goto, Characteristics of a SMA Actuator of Knitted Structure for Artificial Muscle, Transactions of the Japan Society of Mechanical Engineers, Series C, Vol. 71 ,No. 707, 2321-2327 (20057)

[9] J. Abel, J. Luntz, and D. Brei, Smart Materials and Structures, Vol. 21, No. 8, 085011

(Received January 27, 2015; Accepted April 17, 2015) 